\title{
Visualization application of infrared image by the high-speed infrared thermography
}

\author{
T. Yaoita*, F. Yagasakir*, F. Marcotte**
}

*Affiliation 1, Ken Automation, Inc., 1-11-12-5F Hiranuma, Nishi-ku, 220-0023, Yokohama, Japan, emailinfo@kenautomation.com

${ }^{* *}$ Affiliation 2, Telops, Co. Inc., 100-2600 St-Jean-Baptiste Ave, Quebec, G2E6J5, Canada, emailcontact@telops.com

\begin{abstract}
The development of high-performance infrared thermography has given rise to a wide variety of demanding thermal imaging applications that were previously not possible ranging from academics and research, industrial $R \& D$, nondestructive testing and materials testing, aerospace and defence.

\section{Introduction}

The standard infrared thermography has taken certain integration time with the photography per once, it was unsuitable for the high-speed photography. By the infrared thermography which can buffer photography data efficiently continually, high-speed images of 1,000 fps is enables in 640X512 pixels, 3,000 fps in 320X256 pixels and 47,000 fps in $64 \times 50$ pixels (depending on the temperature range) by the windowing mode. The heat generation of specimen phenomenon is used for the monitoring of the start point of the destruction, the temperature measurement of milling cutter, and the thermometry of combustion gases, etc.
\end{abstract}

\section{Experiment}

The first infrared thermography uses cooled InSb infrared detector and it takes enabled 3,000 fps in $320 \times 256$ pixels. Here, I introduce an image example using this high-speed infrared thermography.

\subsection{Impact breaking test of the composite plate}

The target material impacted from the backside of the composite plate (Fig.1). The high-sped infrared thermography took an image in the other side. For the image takes $6,000 \mathrm{fps}$, a composite became the small piece, and a state to be scattered was monitored by the thermography (Fig.2). What about composite becomes the starting point of the destruction? How many prepregs should it repeart to prevent a composite from being broken ? We can find the starting point of the destruction and a direction by heat generation in the high-speed infrared thermography.

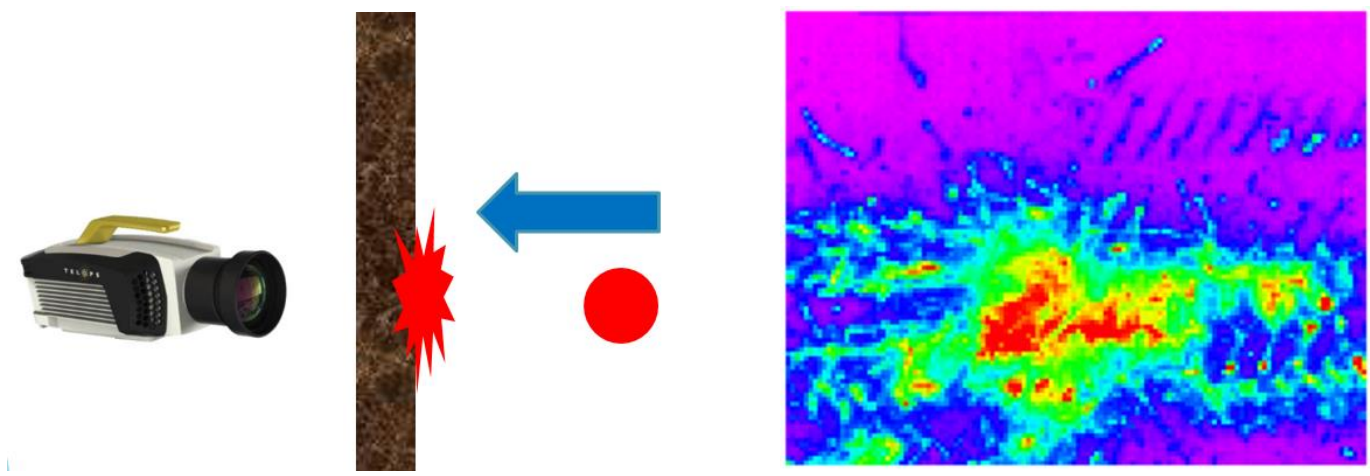

Fig. 1 Impact test of the composite plate

Fig.2 Infrared image of the composite plate during impact test

\subsection{Temperature measurement of the milling cutting tool}

This is a tip of the tungsten carbide adapted on the milling cutting tool. We tried to measure the temperature during milling process using a steel specimen. The milling cutting tool turns in $720 \mathrm{rpm}$ and the frame rate of the infrared thermography is $12,000 \mathrm{fps}$ in $128 \times 64$ pixels (See Fig.3). We set emissivity of the tungsten carbide in 0.05 and acquired the temperature scale (See Fig.4). It is necessary to heat up a tool to a condition same as milling processing, and to decide emissivity of the tool tip exactly. The speed of $12,000 \mathrm{fps}$ is important by this measurement, but greatly varies in the temperature change if one frame of image is different. As the milling cutting tool becomes fragile when the tungsten carbide 
in particular exceeds 800 degrees Celsius. Therefore, the high-speed infrared thermography is useful that it changes the temperature in a very short time.

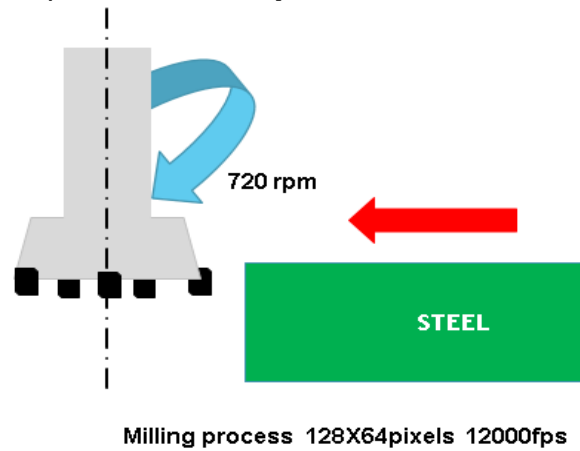

Fig.3 Milling process

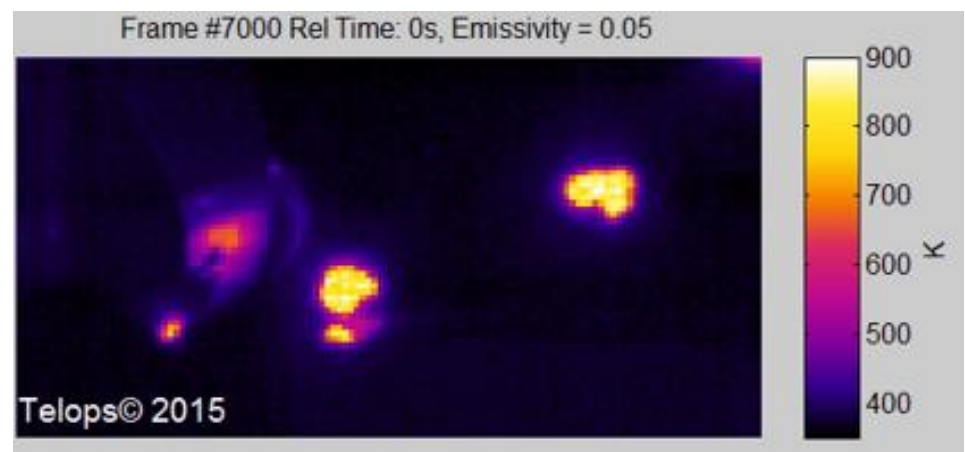

Fig.4 Temperature image of the tungsten carbide tip

\subsection{Development examination of the air bag}

The high-speed infrared thermography can observe the state that the air bag opens, opens starting from where and where and how much high temperature gas leak from it (Fig.5).

And the nylon fiber using with the air bag can penetrate infrared spectrum. It can visualize a flow of the gas in the air bag (Fig.6). As the air bag for the curtain and the air bag for the pedestrian protection are long thinly shape, the identification of the flow of the gas is important. It can be optimized position of these holes to release gas and occasion how to hold up the air bags if it can be identified a flow of the gas. It can be optimized the time when the air bag open and the shape of the air bag, if it can be inspected where is leak of how much gas from it.

It is very difficult to measure temperature of the gas by density, ingredient ratio, real emissivity, and infrared absorption of the gas using the standard infrared thermography. For one method, two infrared wavelengths convert into temperature of the gas.

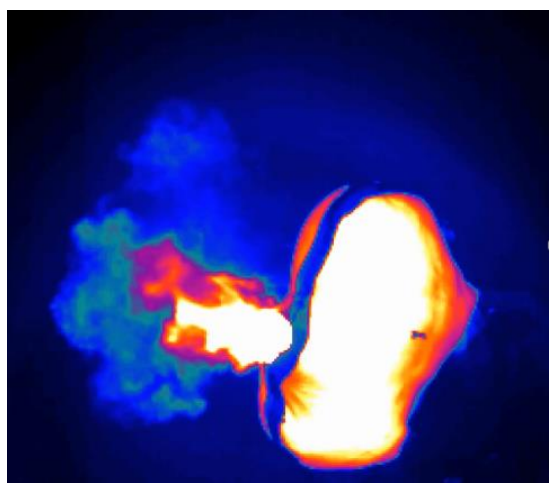

Fig.5 Explosion examination of the air bag

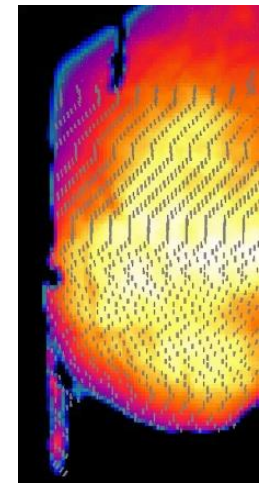

Fig.6 Flow of the gas in the air bag

\section{Summary}

I introduced some application examples using the high-speed infrared thermography. By a technological advance of readout circuit of infrared detector and the performance gain of the PC, high-speed imaging allows even the infrared thermography. The information that the temperature characterizing the infrared thermography in particular relates to is not provided with the visible camera. I think that the infrared camera fits various application from now on if imaging of the high speed is possible with development of the electronic technique more. The heat conduction can evaluate the phenomenon based on the characteristic of materials such as hardness and the depth of hardening of metal. High-speed imaging is important in the materials having high thermal conductivity like metals in particular. In addition, the identification of the gas is possible when the specific band pass filter use absorbing infrared.

\section{REFERENCES}

[1] Visualization of high-speed phenomena using high-speed infrared camera; T. Yaoita, F. Marcotte; The $31^{\text {st }}$ International Congress on High-speed imaging and Photonics, November 7-10, 2016 